\title{
A new technique of epidural and intrathecal catheterization to evaluate pharmacokinetics of epidural administration in dogs: a prospective study
}

Myoung Hoon Kong

Korea University Guro Hospital

Sang Sik Choi ( $\nabla$ clonidine@empal.com )

Korea University Guro Hospital https://orcid.org/0000-0002-0260-9886

Jung Eun Kim

Korea University Guro Hospital

Mi Kyoung Lee

Korea University Guro Hospital

Chung Hun Lee

Korea University Guro Hospital

Yeon Joo Lee

Korea University Guro Hospital

Technical advance

Keywords: Canine; Epidural catheterization; Epidural drug administration; Intrathecal catheterization;

Yaksh's model

Posted Date: April 19th, 2019

DOl: https://doi.org/10.21203/rs.2.9257/v1

License: (c) (i) This work is licensed under a Creative Commons Attribution 4.0 International License.

Read Full License 


\section{Abstract}

Background: Compared to the conventional oral or intravenous drug administration, epidural administration of drugs has significantly higher efficacy and safety. Experimental research on animals should be performed before applying to humans. Unlike the existing canine model, we describe a new and alternative technique of epidural and intrathecal catheterization to investigate the efficacy and safety of epidural drug administration in dogs. Methods: Twelve adult dogs were used in this study. The procedures were performed with dogs in sternal recumbency under deep sedation. Epidural catheterization was performed at the T1-T2 intervertebral space with C-arm fluoroscopy guidance. After confirming loss of resistance, a flexible epidural catheter was passed cranially to the $\mathrm{C} 2-\mathrm{C} 3$ level. Intrathecal catheterization was performed through the cisterna magna with the neck slightly flexed. An 18-gauge Tuohy needle was inserted into the subarachnoid space through the atlanto-occipital space. After confirming cerebrospinal fluid leakage without bleeding, a flexible intrathecal catheter was passed caudally to the $\mathrm{C} 2-\mathrm{C} 3$ level. Results: All epidural and intrathecal catheterizations were successfully performed under deep sedation without any complications. Conclusion: The new technique of epidural and intrathecal catheterization in canines is a safe and alternative procedure for investigating the toxicity and pharmacology of epidurally administered drugs.

\section{Background}

As neuraxial techniques have been extensively used in the past century, considerable progress has been made in epidural drug administration. The epidural technique is not only used commonly in regional anesthesia and postoperative pain control but is also used for the perioperative care of high-risk surgical patients [1-4]. Local anesthetics and opioids are the commonly used epidural agents, and different pharmacological classes of drugs have been investigated for epidural administration. Experimental research on animals should be conducted to determine whether the newly-developed agents can be used safely and effectively in humans and to evaluate their pharmacology and neurotoxicity. A canine model has been developed and used because it shows a response similar to that observed in humans and is comparable to humans in size [5]. Epidural injections in dogs are mostly performed in the lumbosacral space because the space is especially wide in the vertebral structure of dogs [6-10]. In the existing canine model, it was difficult to advance the catheter to a long distance and locate the tip of the intrathecal catheter immediately adjacent to the tip of epidural catheter in order to investigate the pharmacokinetics of a new epidurally administered drug. Hence, we attempted a new technique of inserting the catheter at the upper thoracic $(\mathrm{T} 1-\mathrm{T} 2)$ epidural space under deep sedation.

The present paper describes a new canine model for safe and easy catheterization to investigate the pharmacokinetics of epidurally administered drugs.

\section{Methods}


The study was conducted after obtaining institutional approval for the use of animals by the Korea University Institutional Animal Care and Use Committee (Protocol No. KUIACUC-2011-151).

Twelve adult mongrel dogs with a mean weight of $21 \pm 2.77 \mathrm{~kg}$ were used in this study. Every dog was cared for by well-trained men from the Laboratory Animal Program of the Korea University Medical Center. To prevent distress, experiments were performed in a quiet and comfortable environment; dogs were heavily sedated by intramuscular injection of $0.15 \mathrm{~mL}$ xylazine hydrochloride $\left(50 \mathrm{mg} / \mathrm{kg}\right.$; Rompun ${ }^{\circledR}$ Bayer, Seoul, Korea) and maintained with intramuscular injection of ketamine. Oxygen (100\%) was supplied through a facial mask at a flow rate of $5 \mathrm{~L} / \mathrm{min}$. After sedation, the dog was placed in sternal recumbency on the procedural table in order to carry out the procedure, and the cephalic or accessory cephalic veins were used for fluid administration and blood sampling. The procedures were performed after aseptic preparation.

\section{Epidural Catheterization}

Epidural catheterization was performed at the first thoracic (T1)-second thoracic (T2) intervertebral space with the C-arm fluoroscopy-guided midline approach. Before the procedure, the fur of the lower cervical and upper thoracic areas was shaved and the skin disinfected. The needle entry point was confirmed by palpation of T1 and T2 spinous processes and by C-arm fluoroscopy. An 18-gauge, $90 \mathrm{~mm}$ Tuohy epidural needle (B. Braun Medical, Inc., PA, USA) was inserted in the line between T1 and T2 with the bevel facing cephalad. As the needle was advanced slowly through the supraspinous and interspinous ligaments and pierced the interarcuate ligament, a distinct popping sensation was felt. Then, the epidural space was confirmed by "loss of resistance" test (Fig. 1). A flexible epidural catheter (PerfiX ${ }^{T M}$; B-Brown, Melsungen AG, Germany) was advanced cranially to the second cervical (C2)-third cervical (C3) level through the needle. The location of the catheter tip was verified fluoroscopically by injecting contrast medium into the catheter (Fig. 2). Once the correct position of the catheter was confirmed, it was fixed in place with sterile dressing. We measured the depth from the skin to the epidural space at the T1-T2 level every time. We also measured the length of the epidural catheter inserted at the $\mathrm{C} 2-\mathrm{C} 3$ level.

\section{Intrathecal Catheterization}

Intrathecal catheterization was performed through the cisterna magna (CM) in the sternal recumbency position with the neck slightly flexed. Before the procedure, the fur of the occipital and posterior neck was shaved and the skin disinfected. The needle entry point was the center of a virtual triangle comprising the external protuberance and the two tips of transverse processes of the first cervical vertebra (C1) (Fig. 3). After the bony landmarks were palpated, the 18-gauge Tuohy needle was inserted perpendicularly to the skin in the midline until resistance was no longer felt. After confirming cerebrospinal fluid (CSF) leakage without bleeding, a flexible intrathecal catheter (Perifix; B-Brown, Melsungen AG, Germany) was inserted and advanced caudally to the $\mathrm{C} 2-\mathrm{C} 3$ level so that the tip of the catheter was located immediately adjacent to the tip of the epidural catheter (Fig. 4). The location of the catheter tip was verified fluoroscopically by injecting contrast medium through the catheter (Fig. 2-C). Once the correct position 
was confirmed, the catheter was fixed on the occiput with sterile dressing. We measured the depth from the center of the virtual triangle to the intrathecal space $(\mathrm{cm})$ each time. We also measured the length of the intrathecal catheter inserted at the $\mathrm{C} 2-\mathrm{C} 3$ level.

After acquiring the data, the dogs were euthanized with potassium chloride. After the intramuscular injection of ketamine and xylazine hydrochloride prior to the euthanasia, the dogs were confirmed to be unconscious. Potassium chloride was then intravascularly injected at a dose of $150 \mathrm{mg} / \mathrm{kg}$. Fortunately, clonic spasm did not occur after the administration of potassium chloride. Death of dogs was confirmed by lack of pulse, breathing, corneal reflex, response to a firm toe pinch, and respiratory sounds and heartbeat with stethoscope and by the graying of the mucous membranes and rigor mortis. The euthanasia methods employed in this study followed the American Veterinary Medical Association (AVMA) guidelines for the euthanasia of dogs.

\section{Results}

Initially, we performed epidural catheterization through the lumbosacral area to assess the pharmacokinetics of new agents administered epidurally, which is mainly used in the existing canine model. However, it was difficult to advance the catheter to place the tips of the epidural and intrathecal catheters at the level of the vertebrae. Hence, we attempted to perform epidural catheterization at the upper thoracic level as soon as possible. We safely and easily performed epidural catheterization at T1T2 and intrathecal catheterization at CM and located it at the expected level (C2-C3) under deep sedation in the twelve dogs. The depth from the skin to the epidural space at T1-T2 level was $7.64 \pm 0.63 \mathrm{~cm}$ (mean \pm SD) and the depth from the center of the virtual triangle to the intrathecal space was $3.16 \pm 0.47$ $\mathrm{cm}$ (mean $\pm \mathrm{SD}$ ). The tips of epidural and intrathecal catheter were successfully located at the $\mathrm{C} 2-\mathrm{C} 3$ intervertebral space level. The length of the epidural catheter from the T1-T2 to the $\mathrm{C} 2-\mathrm{C} 3$ intervertebral space was $19.36 \pm 2.58 \mathrm{~cm}$ (mean $\pm \mathrm{SD}$ ). The length of the intrathecal catheter from the $\mathrm{CM}$ to the $\mathrm{C} 2-$ C3 intervertebral space was $5.59 \pm 1.86 \mathrm{~cm}$ (mean $\pm \mathrm{SD}$ ). We could obtain sufficient CSF samples from the intrathecal catheter.

\section{Discussion}

We considered that the neuraxial administration of antiviral agents to the spinal structures with the highest viral burden might be more effective than the other routes of drug administration in herpes zoster and conducted the study to observe neurological safety after an intrathecal acyclovir injection in rats. There was no evidence of neurological and histopathological abnormality following intrathecal acyclovir injection in rats [11]. We also compared intrathecal concentrations of acyclovir following epidural and intravenous (IV) administration in rats to investigate the pharmacokinetics of acyclovir. We found that the epidural administration of acyclovir provided superior drug concentration in the intrathecal space compared with IV administration [12]. We thought that further experimental research using other animal species should be conducted to evaluate the safety, efficacy, and toxicity of acyclovir before clinical application in humans. Hence, we decided to use the canine model because it showed a response similar 
to that observed in humans and is comparable in size [5]. We performed epidural catheterization through the lumbosacral area using the existing canine model to assess the pharmacokinetics of epidurally administered acyclovir [8]. However, this technique was time-consuming, and it was difficult for us to advance the catheter a long distance in order to place the tips of the epidural catheter and intrathecal catheter at the level of the vertebrae. Heath et al. reported that analgesia was absent in $12 \%$ of dogs in which lumbosacral epidural analgesia technique was attempted [13]. One of the major complications of epidural analgesia in dogs and cats is technical failure to locate the lumbosacral space and/or insert a needle into the epidural space, but reports of individual technical failures are rare [13]. Epidural catheterization of the cervical spine of the dog is difficult because the superior lamina overrides the inferior lamina, creating a small interlaminar space. In contrast, epidural catheterization of the thoracic spine is not difficult since the lamina does not cover the intervertebral space [14]. It is also easy to detect the spinous processes of the thoracic vertebrae by palpation because they are considerably longer than those in the cervical vertebrae. Based on the anatomy of the canine spine, we chose to insert the Touhy needle at the T1-T2 intervertebral space. The needle was easily inserted into the epidural space using the "loss of resistance" technique and advanced to the C2-C3 level $(19.36 \pm 2.58 \mathrm{~cm})$. Similar to Yaksh's model, intrathecal catheterization was performed between the foramen magnum and the first cervical vertebra (C1); however, we performed the procedure using a Touhy needle without dissection, and the catheter was caudally advanced to the $\mathrm{C} 2-\mathrm{C} 3$ level $(5.59 \pm 1.86 \mathrm{~cm})$. In this way, we were able to easily advance the catheter to the desired spinal level epidurally and intrathecally. Then, the catheter was maintained firmly in place. This technique was less stressful for the dogs because of the noninvasiveness of the procedures. Currently, we are conducting a canine experiment to investigate the pharmacokinetics of epidurally administered acyclovir using the new technique. We expect this technique to be useful in surgery or pain control in the forelimb and upper part of dogs.

\section{Conclusions}

The new technique of epidural and intrathecal catheterization used in dogs in this study is a safe and alternative procedure for studying the toxicity and pharmacology of epidurally administered drugs.

\section{Abbreviations}

C: cervical

T: thoracic

CM: cisterna magna

CSF: cerebrospinal fluid

IV: intravenous

\section{Declarations}




\section{Ethics approval and consent to participate:}

The study was conducted after institutional approval for the use of animals by the Korea University Institutional Animal Care and Use Committee (Protocol No. KUIACUC-2011-151).

\section{Consent for publication:}

Not applicable

\section{Availability of data and material:}

The data analyzed during the current study are available from the corresponding author upon reasonable request.

\section{Competing interests:}

The authors declare that they have no conflicts of interest.

\section{Funding:}

This research was funded by College of Medicine, Korea University. However, the funder did not have any additional role in the study design, data collection and analysis, decision to publish, or preparation of the manuscript.

\section{Author's Contributions:}

MKL and SSC designed the experiments. MHK, JEK, MKL, CHL, YJL, and SSC performed the experiments. MHK and SSC wrote the manuscript. All authors contributed to editing the manuscript.

\section{Acknowledgements:}

Not applicable

\section{References}

1. Liu S, Carpenter R, Neal JM. Epidural anesthesia and analgesia. Their role in postoperative outcome. Anesthesiology. 1995;82:1474-506.

2. Negre I, Gueneron JP, Jamali SJ, Monin S, Ecoffey C. Preoperative analgesia with epidural morphine. Anesth Analg. 1994;79:298-302.

3. Benzon HT, wong HY, Belavic AM Jr, Goodman I, Mitchell D, Lefheit T, et al. A randomized doubleblind comparison of epidural fentanyl infusion versus patient controlled analgesia with morphine for postthoracotomy pain. Anesth Analg. 1992;76:316-22.

4. Hjortso NC, Neumann P, Frosig F, Andersen T, Lindhard A, Rogon E, et al. A controlled study on the effect of epidural analgesia with local anesthetics and morphine on morbidity after abdominal 
surgery. Acta Anaesthesiol Scand. 1985;29:790-6.

5. Lebeaux MI. Experimental epidural anaesthesia in the dog with lignocaine and bupivacaine. $\mathrm{Br} \mathrm{J}$ Anaesth. 1973;45:549-55.

6. Durant PA, Yaksh TL. Distribution in cerebrospinal fluid, blood, and lymph of epidurally injected morphine and inulin in dogs. Anesth Analg. 1986;65:583-92.

7. Bednarski R, Grimm K, Harvey R, Lukasik VM, Penn WS, Sargent B, et al. AAHA anesthesia guidelines for dogs and cats. J Am Anim Hosp Assoc. 2011;47:377-85.

8. Jones RS. Epidural analgesia in the dog and cat. Vet J. 2001;161:123-31.

9. Benson GJ, Thurmon JC. Anesthesia for cesarean section in the dog and cat. Mod Vet Pract. 1984;65:29-32.

10. Klide AM, Soma LR. Epidural analgesia in the dog and cat. J Am Vet Med Assoc. 1968;153:165-73.

11. Kim H, Choi YL, Lee DK, Choi SS, Lee IO, Kong MH, et al. The neurological safety of intrathecal acyclovir in rats. Pain Physician. 2012;15:E107-13.

12. Kim JH, Lee MK, Kim JE, Kim SH, Choi SS. Comparison of intrathecal concentrations of acyclovir following epidural and intravenous administration in rats. Pain Physician. 2016;19:E613-19.

13. Heath RB, Broadstone RV, Wright M, Grandy JL. Using bupivacaine hydrochloride for lumbosacral epidural analgesia. Compend Cont Educ Pract Vet. 1989;11:50-55.

14. Evans H, Lahunta A. Miller's anatomy of the dog. $4^{\text {th }}$ edition. St. Louis: Elsevier; 2013. p.113-21.

\section{Figures}

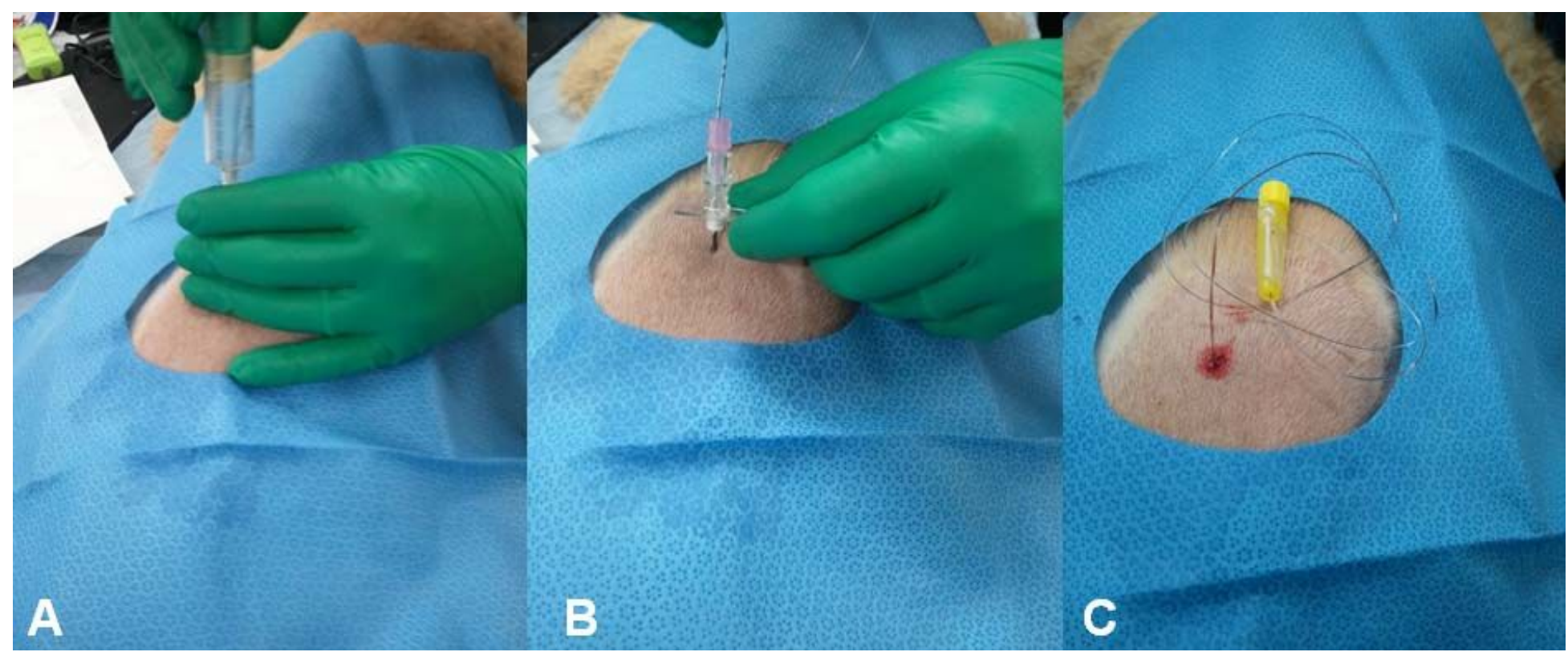

Figure 1 
Thoracic epidural catheterization in a dog [A] Epidural catheterization was performed at the midline of the T1-T2 intervertebral space using an 18-gauge Tuohy needle $[B, C]$. The epidural catheter was inserted through the needle after the presence of the needle in the epidural space was confirmed by the loss of resistance, and the needle was advanced cranially to the $\mathrm{C} 2-\mathrm{C} 3$ level.

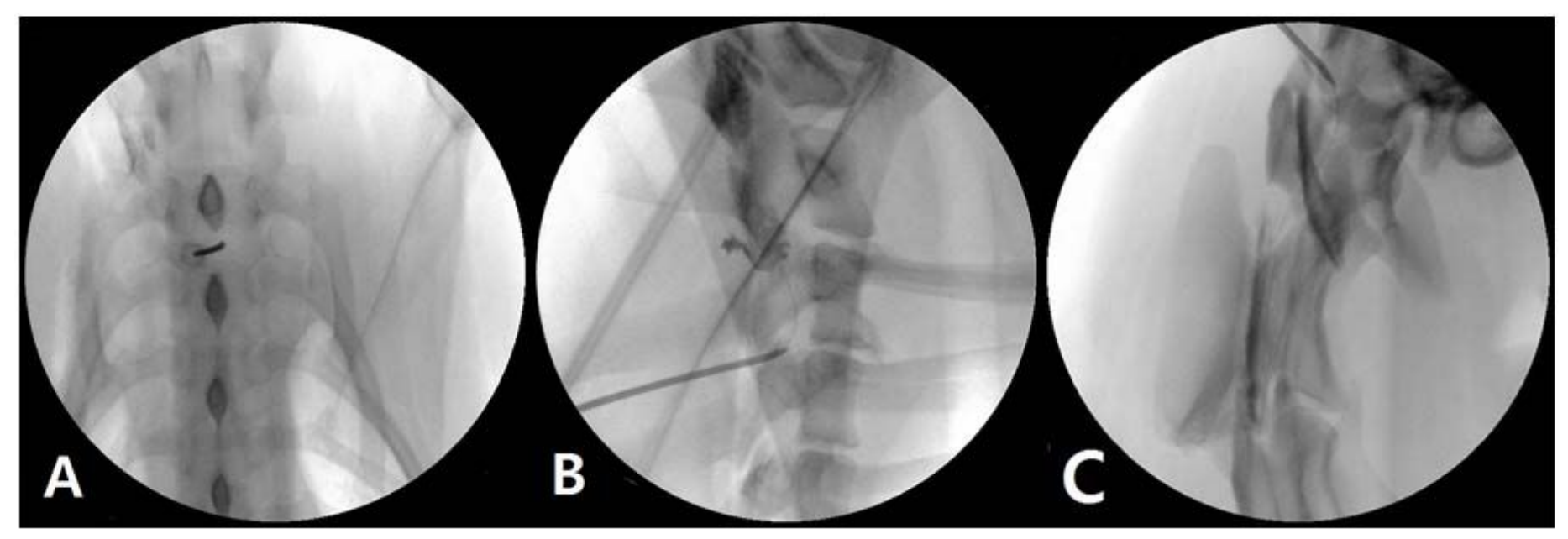

Figure 2

Anteroposterior (A) and lateral (B, C) fluoroscopic images [A] Anteroposterior fluoroscopic image. The Tuohy needle position was confirmed at the level of T1-T2 intervertebral space by the fluoroscopic image. $[\mathrm{B}]$ The tip of the epidural catheter was advanced cranially to the $\mathrm{C} 2-\mathrm{C} 3$ level in the lateral fluoroscopic image. [C] The intrathecal catheter was progressed caudally to the C2-C3 level (white empty triangle). 


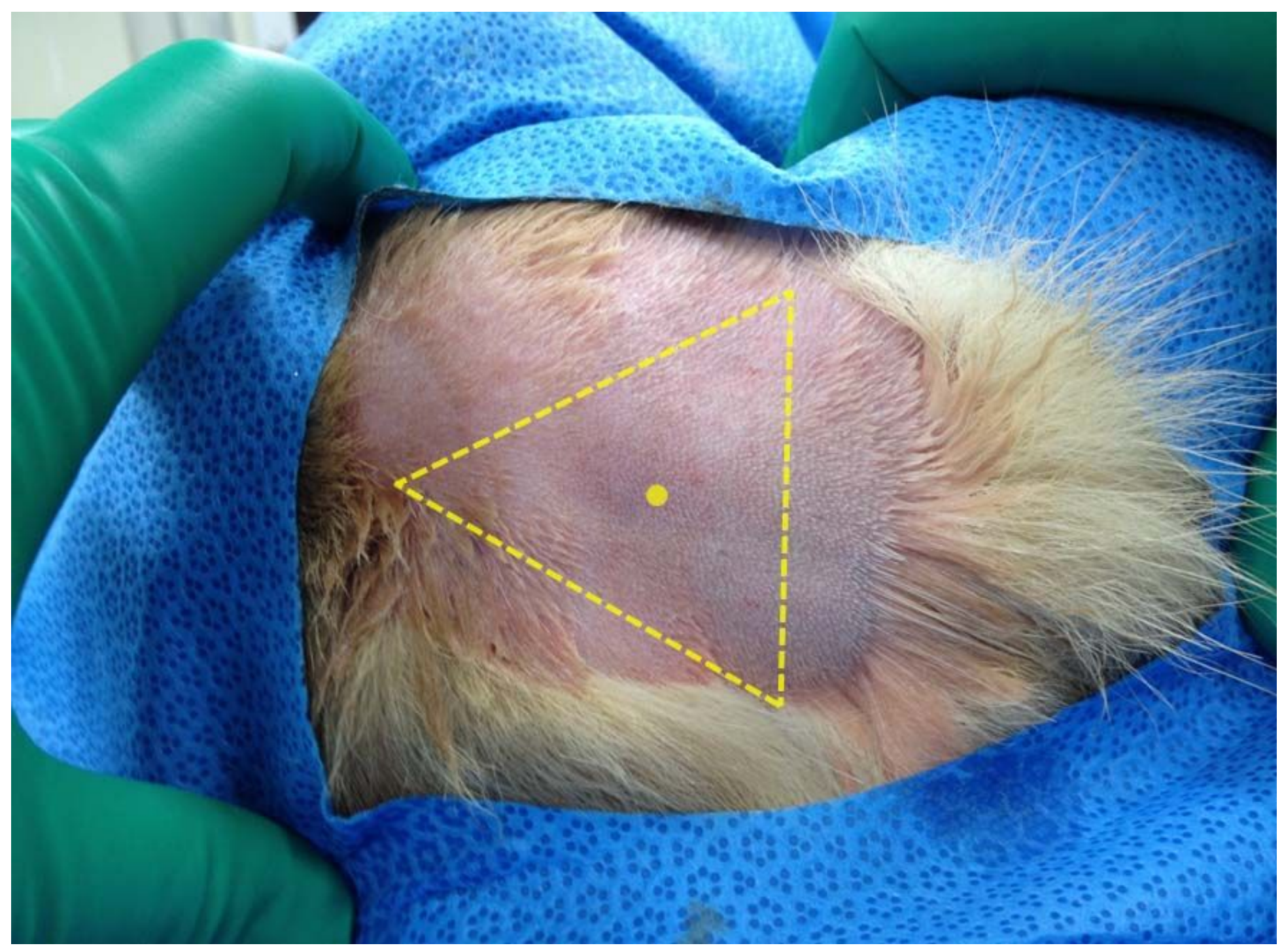

Figure 3

Virtual triangle comprising external protuberance and tips of transverse processes of the first cervical vertebra. The external occipital protuberance and both tips of the transverse processes of $\mathrm{C} 1$ were located using palpation; these three points formed the vertices of a triangle. The center of a triangle was the needle entry point for intrathecal catheterization. An 18-gauge Tuohy needle was inserted into the cisterna magna (CM). 


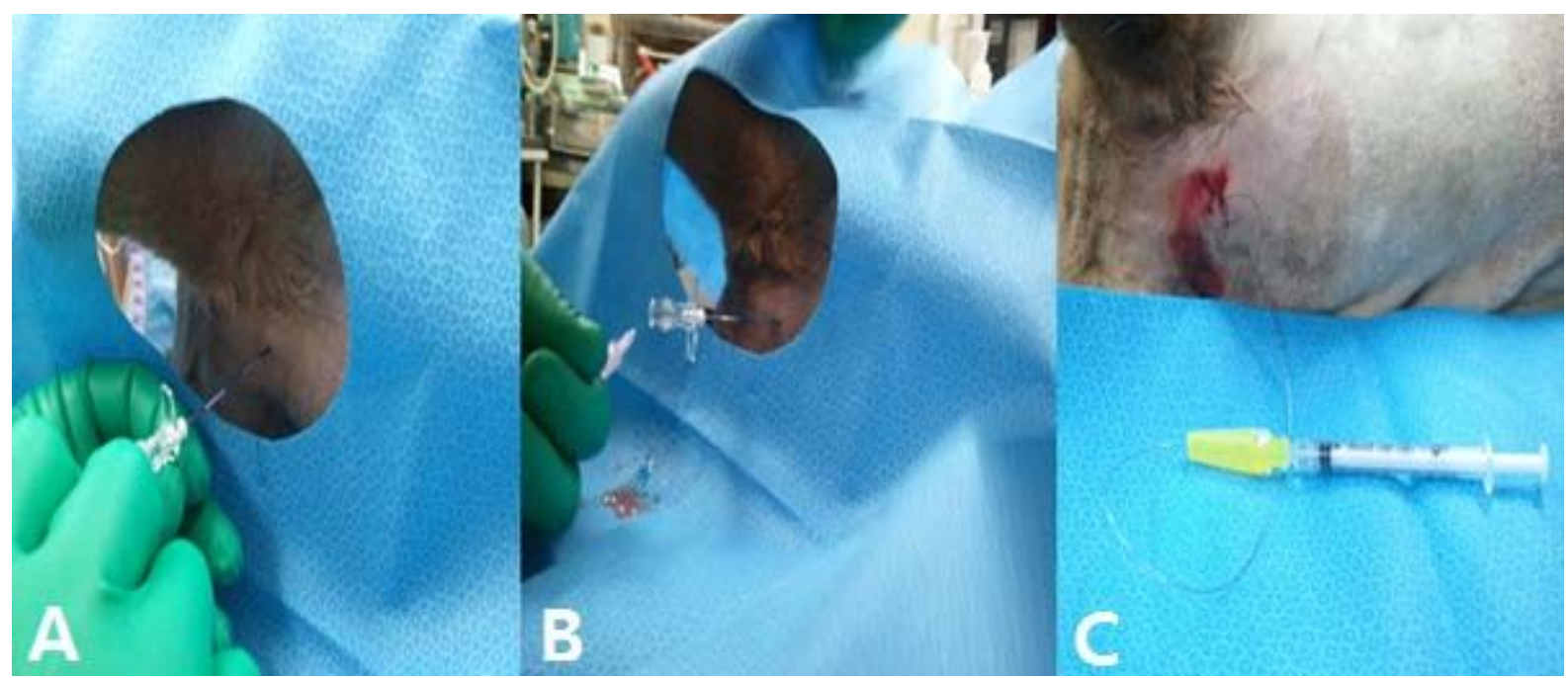

Figure 4

Intrathecal catheterization in a dog. [A] The needle was inserted into the center of the triangle, perpendicularly to the plane of the triangle checking the midline. [B] After confirming the presence of CSF, the intrathecal catheter was inserted caudally at the $\mathrm{C} 2-\mathrm{C} 3$ level through the needle with the bevel facing caudad. [C] One milliliter of cerebrospinal fluid was obtained to confirm patency of the catheter.

\section{Supplementary Files}

This is a list of supplementary files associated with this preprint. Click to download.

- supplement1.xIsx

- supplement2.docx 